\title{
Interprétation et décodage argumentatifs
}

Argumentative interpretation and decoding

\section{Marion Carel}

Escola de Altos Estudos em Ciências Sociais - EHESS - Paris - França

Résumé: La lecture n'est pas seulement affaire de décodage, c'est là une banalité : faut-il encore percevoir les sous-entendus, communiqués par les énoncés au delà de ce qu'ils disent; faut-il encore comprendre les allusions intertextuelles, les intentions de l'auteur; faut-il encore remarquer le style du texte, sa complexité grammaticale, ses allitérations - j'en oublie. Ce que je voudrais montrer ici, c'est que, même en se limitant à la question du sens immédiat des énoncés, la compréhension d'un texte n'est pas seulement affaire de décodage. La langue nous contraint à interpréter, et cela dès le niveau de ce que dit l'énoncé. Le texte n'est alors, ni simplement à comprendre à partir de la signification de ses mots pleins, ni à compléter comme les textes à trou de l'école qui permettent de vérifier que l'interlocuteur partage le même savoir que le locuteur. Le locuteur n'impose pas totalement le sens de l'assemblage de ses mots et le lecteur doit inventer en partie ce sens.

Mots-clés: Lecture. Argumentation. Interprtation. Dcodage.

Abstract: Reading is not only a matter of decoding, this is a commonplace: it is also necessary to realize the implicit information communicated by the statements beyond what is written; furthermore, it requires understanding the intertextual references, the author's intentions; and moreover, the style of the text must be observed, its grammatical complexity, its alliterations - I forget that. What is intended to be shown is that, even if limited to the immediate meaning of statements, text comprehension is more than a decoding task. The language forces an interpretation from the level of what is said in the statement. Therefore, the text is not supposed to be comprehended simply through the meaning of its full words, neither to be completed, such as in gapfill tasks at school, which enables to verify that the interlocutor shares the same knowledge as the writer. The writer does not completely impose the meaning of the words set, and the reader should create part of this meaning.

Keywords: Reading. Argumentation. Interpretation. Decoding. 
La lecture n'est pas seulement affaire de décodage, c'est là une banalité: faut-il encore percevoir les sous-entendus, communiqués par les énoncés au delà de ce qu'ils disent; faut-il encore comprendre les allusions intertextuelles, les intentions de l'auteur ; faut-il encore remarquer le style du texte, sa complexité grammaticale, ses allitérations - j'en oublie. Ce que je voudrais montrer ici, c'est que, même en se limitant à la question du sens immédiat des énoncés, la compréhension d'un texte n'est pas seulement affaire de décodage. La langue nous contraint à interpréter, et cela dès le niveau de ce que dit l'énoncé. Le texte n'est alors, ni simplement à comprendre à partir de la signification de ses mots pleins, ni à compléter comme les textes à trou de l'école qui permettent de vérifier que l'interlocuteur partage le même savoir que le locuteur. Le locuteur n'impose pas totalement le sens de l'assemblage de ses mots et le lecteur doit inventer en partie ce sens. Cela se produit en poésie («Marche », Poèmes.19111918, G. Chennevière) :

\section{Qu'il fait gris ! Qu'il fait froid !}

Une monotone pluie

A cousu le ciel aux toits.

Je pense aux pieds nus d'un enfant qui dort.

Que signifie en effet que le ciel est cousu aux toits ? Qu'il n'y a pas de perspective, que la vue s'arrête tout de suite, ou plutôt que la nature est abimée, brimée, détruite? On retrouve la même liberté interprétative dans la publicité :

Tous ces petits accessoires au design très reconnaissable et à la couleur jaune vif donnent du peps à votre quotidien et à votre intérieur

En quoi le jaune vif donne de l'énergie à mon quotidien - ou à mon intérieur ? Parce qu'il est vif ? Parce qu'il est d'une couleur inattendue? Et qu'en est-il du rôle du design reconnaissable? Même interrogation encore, et même liberté interprétative, dans ce passage du très raisonnable journal français Le Monde - l'article est à propos d'une conférence de presse donnée la veille par le Président de la République Française:
Le président veut renouer avec les corps intermédiaires, qui se sont sentis négligés sur le début de son quinquennat.

Le président réagirait-il au «sentiment » des syndicats à la manière, toute XIXème siècle, d'un amant face à une maîtresse qu'il délaisse ? Ou doiton comprendre qu'il répond à une colère? Une menace ? Là encore, le lecteur doit inventer.

Après une première partie présentant le phénomène de décodage à l'intérieur de la perspective argumentative qui est la mienne, je présenterai, en réponse, le phénomène d'interprétation que je viens d'évoquer. II apparaîtra que l'interprétation, pour libre qu'elle soit, est tout de même guidée par la langue: plusieurs chemins existent, mais à l'intérieur d'une carte tout à fait précise. Je terminerai l'article par l'étude d'exemples liés, non plus au sens des énoncés, mais à la signification linguistique des mots eux-mêmes. Je reviendrai ainsi, une fois encore, sur mais, de manière à montrer que l'interprétation est un procédé sémantique, déterminant ce qui est dit et non ce qui est sous-entendu. L'interprétation, comme l'argumentation, est dans la langue.

\section{Le décodage argumentatif}

La Théorie des Blocs Sémantiques (Carel 2017), à la fois cadre et objet de mes recherches, a pour slogan que tout énoncé est paraphrasable par des enchaînements argumentatifs, qu'il s'agisse d'enchaînements comportant une conjonction du type de donc ou d'enchaînements comportant une conjonction du type de pourtant. Ainsi, l'énoncé (1) est paraphrasable par (2):

(1) le chat voyait la souris approcher

(2) le chat regardait la souris et donc savait qu'elle approchait

et l'énoncé (3) - je reprends son étude à Saenz Suarez (2019) - par l'enchaînement argumentatif (4):

(3) le spectacle que nous sommes allés voir était mauvais 
(4) nous avons regardé des actions se dérouler et pourtant nous n'avons pas été émus

De l'énoncé à l'enchaînement, il y a décodage argumentatif.

Pour développer cela, convenons d'abord de distinguer à l'intérieur d'un enchaînement argumentatif des termes «fondateurs » et des termes qui ne le sont pas. Les termes fondateurs de (2) sont regarder et savoir. Ce sont ces verbes que donc relie argumentativement et, parce qu'ils sont à nouveau reliés par si à l'intérieur de (5), ce dernier enchaînement apparaît semblable à (2):

(5) si la chienne regarde le chat, elle saura qu'il s'éloigne

Tous les deux partagent une même structure, ils sont construits sur un même schéma : je le noterai REGARDER DC SAVOIR et je le qualifierai de schéma «fondateur » de (2) et de (5) (le DC rappelle que les enchaînements (2) et (5) qui donnent réalité à ce schéma comportent eux-mêmes une conjonction du type de donc, que cela soit la conjonction donc ellemême, ou la conjonction si). De manière parallèle, l'enchaînement (4) a pour termes fondateurs regarder et ne pas être ému; il concrétise un schéma que je noterai REGARDER PT NEG ÊTRE ÉMU, et qui constitue également le squelette de (6):

(6) même s'il regarde ce qui se passe autour de lui, il ne sera pas ému

- le symbole PT dans le nom du schéma rappelle que les enchaînements qui le concrétisent comportent une conjonction du type de pourtant, qu'il s'agisse de pourtant ou de même si.

Revenons alors au décodage de (1) et de (3). Comment est évoqué (2) ? Je ferai l'hypothèse que la signification du verbe voir contient le schéma REGARDER DC SAVOIR: voir est une manière de regarder qui aboutit à un savoir. Son emploi dans le chat voyait la souris approcher signale alors que la paraphrase argumentative de cet énoncé a pour structure REGARDER DC SAVOIR, les emplois de le chat et de la souris approcher donnant chair à cette structure en déterminant les termes non fondateurs de la paraphrase argumentative. Je dirai que l'emploi du verbe voir est «constitutif ». II exprime sa signification, l'impose comme structure de la paraphrase et détermine les termes fondateurs de la paraphrase argumentative.

Le décodage argumentatif de (3), ainsi que le rôle de son terme constitutif, sont un peu différents. Cette fois, c'est le nom spectacle, présent dans le sujet grammatical de (3), qui est constitutif. II signifie le schéma REGARDER DES ACTIONS DC ÊTRE ÉMU (un spectacle est regardé, et cela lui permet de produire des sentiments chez celui qui le regarde), puis ce schéma est transformé en REGARDER DES ACTIONS PT NEG ÊTRE ÉMU par l'adjectif attribut mauvais: un mauvais spectacle n'est pas tout à fait un spectacle, certaines des caractéristiques du spectacle sont transformées, un mauvais spectacle n'est pas émouvant. C'est ce schéma transformé qui constitue la structure de l'enchaînement évoqué par (3). La relative que nous sommes allés voir en détermine le terme non fondateur nous et le temps grammatical. Est ainsi évoqué (4) nous avons regardé des actions se dérouler et pourtant nous n'avons pas été émus.

Un terme constitutif ne fournit donc pas nécessairement la structure de la paraphrase. II signifie un premier schéma qui peut ensuite être transformé par un «opérateur » - c'est là le rôle de l'emploi de mauvais dans (3). C'est seulement le schéma transformé qui fournit la structure de la paraphrase argumentative.

Un troisième cas est celui du décodage argumentatif de (7). Nous allons voir cette fois que le terme constitutif ne détermine pas nécessairement les termes fondateurs de l'enchaînement évoqué, mais se contente d'en préfigurer le sens:

(7) Pierre a eu la prudence de rentrer avant la pluie

(7) communique en effet que Pierre est rentré à cause de l'arrivée de la pluie, raison qui disparaît si on remplace prudence par chance: le locuteur de Pierre a eu la chance de rentrer avant la pluie communiquerait que Pierre a simplement fait l'action de rentrer ; il se serait trouvé par ailleurs que c'était avant la pluie. Je rendrai compte du rôle de prudence dans (7) en disant que son emploi est constitutif. II 
exprime sa signification, à savoir le schéma DANGER DC PRÉCAUTION (être prudent, c'est prendre des précautions à cause du danger) et ce schéma est le squelette de l'enchaînement qui paraphrasera (7) - (7) ne comporte pas d'opérateur.

Une différence apparaît cependant avec (1). Car l'énoncé (7) comporte également les termes avant la pluie et rentrer qui spécifient respectivement le danger en question et la précaution prise par Pierre. (7) évoque ainsi, non pas simplement (8) dans lequel les circonstances de la prudence de Pierre disparaîtraient -, mais bien (9):

(8) Pierre a rencontré un danger donc il a pris des précautions

(9) il allait pleuvoir et donc Pierre est rentré

On comprend la différence avec (1). Tandis que, dans (1), le terme constitutif voir impose les termes fondateurs de la paraphrase argumentative, par contre, dans (7), le terme constitutif se contente de donner le sens des termes fondateurs: le terme prudence fait voir la pluie comme un danger et le fait de rentrer comme une précaution.

Cette possibilité m'amène à une précision. J'ai dit que, selon la Théorie des Blocs Sémantiques, tout énoncé est paraphrasable par des enchaînements argumentatifs. C'est un sens fort que je donne à «paraphrasable». Ce que je soutiens, c'est que les enchaînements argumentatifs évoqués par l'énoncé étudié représentent complètement son sens. Or la seule donnée d'un enchaînement, sans précision du schéma concrétisé, peut constituter une perte de sens. Ainsi (10) et (11) évoquent tous les deux (12), sans être pour autant sémantiquement équivalents:

(10) Pierre a courageusement pris la parole devant tout le monde

(11) Pierre a audacieusement pris la parole devant tout le monde

(12) tout le monde était assemblé pourtant Pierre a pris la parole

Dans les deux cas la lecture s'effectue par décodage argumentatif. L'adverbe est constitutif, contrairement à ce que son statut grammatical de complément « circonstanciel » pourrait laisser penser. L'adverbe est constitutif, mais sans déterminer les termes fondateurs de la paraphrase: ce sont les termes devant tout le monde et prendre la parole, que l'on trouve aussi bien dans (10) que dans (11), qui ont cette fonction. (10) et (11) évoquent ainsi tous les deux (12). Or (10) et (11) ne sont pas équivalents sémantiquement. L'adverbe courageusement signifie NEG ENVIE PT FAIT et devant tout le monde décrit dans (10) une situation dans laquelle, selon le locuteur, Pierre n'avait pas envie de parler. Par contre audacieusement signifie RISQUÉ PT FAIT et devant tout le monde décrit dans (11) une situation dans laquelle, selon le locuteur, il est risqué de parler. Pour rendre compte de cette différence entre (10) et (11) je préciserai que (10) évoque (12) compris comme concrétisant NEG ENVIE PT FAIT, tandis que (11) évoque (12) compris comme concrétisant RISQUÉ PT FAIT.

Autrement dit, le slogan de la Théorie des Blocs Sémantiques doit être un peu compliqué. II ne s'agit pas seulement de dire que tout énoncé est paraphrasable par des enchaînements argumentatifs, mais que tout énoncé est paraphrasable par des enchaînements argumentatifs attachés aux schémas qu'ils concrétisent. Trouver le sens d'un énoncé, c'est être capable à la fois de le paraphraser par un enchaînement argumentatif et d'attacher cet enchaînement au schéma qu'il concrétise. Dans ce cadre, un terme constitutif est un terme dont la signification exprime l'aspect qui sera concrétisé éventuellement après transformation par un opérateur. Dans le cas des énoncés (1) et (3), les termes constitutifs fournissent de plus les termes fondateurs des enchaînements évoqués. Mais tel n'est pas toujours le cas, comme le montrent les énoncés (7), (10) et (11), dont les termes constitutifs ont pour seul rôle de donner le sens des termes fondateurs des enchaînements évoqués. Décoder argumentativement un énoncé, c'est déterminer le terme constitutif et donner à la signification de ce dernier le rôle d'encadrer la paraphrase argumentative.

Une dernière remarque, relative au nombre d'enchaînements évoqués par un même énoncé. J'ai jusqu'ici fait correspondre, à chaque énoncé, un seul 
enchaînement. C'est simplificateur. Les termes comportent en fait plusieurs schémas dans leur signification et leur emploi constitutif donne lieu à l'évocation de plusieurs enchaînements. Ainsi courageusement signifie NEG ENVIE PT FAIT mais également BIEN DC FAIT, de sorte que (10) évoque, non seulement (12), mais aussi (13):

(13) prendre la parole était bien donc Pierre l'a fait, compris comme concrétisant BIEN DC FAIT

On peut représenter cela par le schéma suivant:

de cette manière (12) et (13), ainsi que Pierre a été courageux donc il a été admiré ou Pierre a été courageux pourtant il n'a pas été admiré. Le terme constitutif présente comme un tout ces diverses facettes de son sens.

En conclusion, le décodage argumentatif suppose donc que l'énoncé comporte un terme constitutif (voir, spectacle, prudence, courageusement, audacieusement dans nos exemples). Ce terme exprime sa signification argumentative et les schémas communiqués -

manquer d'envie

NEG ENVIE PT FAIT

(12)

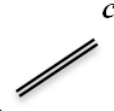

courageusement

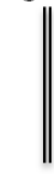

BIEN DC FAIT

(13)

II s'agit là d'une région de la Carte Argumentative du Lexique dans laquelle les mots sont reliés par l'intermédiaire des schémas argumentatifs qu'ils signifient ou préfigurent. La double barre indique que le mot signifie, au sens strict, le schéma : les schémas NEG ENVIE PT FAIT et BIEN DC FAIT appartiennent à la signification de courageusement (ainsi qu'à celle de courage ou de courageuse, que je ne distingue pas). Les barres simples indiquent que le mot «préfigure » seulement le schéma. En effet, on n'est pas nécessairement admiré parce qu'on est courageux ; on peut également être méprisé bien qu'on soit courageux. Autrement dit, certains emplois de courageusement expriment le schéma COURAGEUX DC ADMIRÉ ; tandis que d'autres expriment COURAGEUX PT NEG ADMIRÉ. C'est cette alternative qui est présente dans la signification de courageusement, et non chacun des deux schémas. C'est pourquoi je dis que courageusement «préfigure » - préfigure et non signifie - COURAGEUX DC ADMIRÉ ; de même admiré «préfigure » COURAGEUX DC ADMIRÉ et manquer d'envie «préfigure » NEG ENVIE PT FAIT. La lecture de (10) s'effectue par un décodage argumentatif en cela que le lecteur parcourt la Carte Argumentative du Lexique en allant du terme constitutif courageusement vers les schémas signifiés et préfigurés. (10) évoque après éventuellement transformation fournissent le sens des enchaînements évoqués. L'énoncé évoque ainsi une pluralité d'enchaînements regroupés, en quelque sorte, en un même bouquet, un même " complexe argumentatif». Le terme constitutif a cette double fonction d'exprimer les schémas des enchaînements évoqués et de regrouper leur pluralité à l'intérieur d'un unique complexe argumentatif. Des recherches restent à faire dans le cadre de la Théorie des Blocs Sémantiques pour découvrir les règles de détermination du terme constitutif. Citons dans cette direction les travaux précurseurs de Lescano (2005 et 2008).

Mais que se passe-t-il lorsque l'énoncé ne comporte pas de terme constitutif?

\section{L'interprétation}

Mais comment trouver le sens de le fait d'être regardée fit rougir Mme Hermet? Certes cet énoncé évoque l'enchaînement Mme Hermet était regardée et donc elle rougit, mais quel schéma concrétise cet enchaînement? Doit-on comprendre que Mme Hermet manifeste du plaisir, du déplaisir, simplement une émotion? Avec quels autres enchaînements doit-il être regroupé, dans quel complexe argumentatif faut-il le placer? Doit-on comprendre que Mme Hermet gardera un bon souvenir de l'événement? Un mauvais souvenir?

Une précision tout de suite, relative, au delà des énoncés, à la structure textuelle elle-même. 
Comme le montre Christopulos (2018), un texte est organisé en «périodes argumentatives » coordonnant tous les énoncés qui développent la signification d'un même terme. Ainsi, le paragraphe suivant, qui contient trois énoncés, ne constitue qu'une seule période argumentative, organisée par le terme sensiblerie:

(14) Tu devrais te méfier de tes jugements. Je trouve que tu fais parfois preuve de sensiblerie. Même ce roman stéréotypé te fait pleurer.

Le troisième énoncé évoque l'enchaînement argumentatif (15):

(15) ce roman est stéréotypé pourtant il te fait pleurer

II ne comporte pas lui-même de terme constitutif mais il appartient à une période organisée par le terme sensibleriequi va déterminer, par décodage argumentatif, les schémas concrétisés par tous les énoncés de la période et regrouper les enchaînements évoqués en un même complexe argumentatif. Ainsi, l'enchaînement (15) sera attaché au schéma NEG ÉMOUVANT PT ÉMU signifié par sensiblerie; puis l'énoncé tu devrais te méfier de tes jugements sera paraphrasé par l'enchaînement (16):

(16) tu fais parfois preuve de sensiblerie donc tu devrais te méfier de tes jugements

- puisque la signification de sensiblerie préfigure également le schéma SUJET À LA SENSIBLERIE DC JUGE MAL ; et enfin (15) et (16) seront regroupés à l'intérieur d'un même complexe argumentatif. II y a décodage argumentatif. On peut le représenter sur la figure suivante:

La lecture de (14) s'effectue comme la lecture de (10).

ému, pleurer

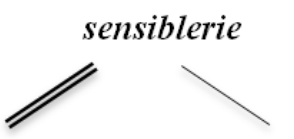

NEG EMOUVANT PT EMU

SUJET A SENSIBLERIE DC JUGE MAL

Le lecteur parcourt la Carte Argumentative du Lexique en allant du terme sensiblerie vers les schémas signifiés ou préfigurés. Le terme sensiblerie est constitutif et détermine que l'enchaînement (15) concrétise NEG ÉMOUVANT PT ÉMU et appartient au même complexe argumentatif que l'enchaînement (16). Un terme peut diriger le décodage argumentatif d'un énoncé sans lui appartenir matériellement.

Je simplifiais donc en m'inquiétant de l'absence de terme constitutif à l'intérieur de l'énoncé le fait d'être regardée fit rougir Mme Hermet: ce qu'il faut considérer, ce sont les cas où manque le terme consitutif à l'intérieur de la période argumentative dans laquelle l'énoncé prend place. Or cela se produit, comme nous allons le voir sur quelques exemples. Nul décodage alors, puisqu'aucun terme n'est matériellement là pour exprimer sa signification, et permettre d'attacher l'enchaînement à un schéma et le placer parmi d'autres enchaînements. Le lecteur doit inventer: je dirai «interpréter argumentativement ». Nous allons voir qu'en partie libre (puisque le locuteur ne formule pas explicitement de terme constitutif), l'interprétation argumentative est cependant guidée par deux règles: mobiliser des schémas doxaux et suivre, à nouveau, la Carte Argumentative du Lexique.

Commençons par la question des schémas concrétisés, et par l'exemple, déjà cité, de la strophe de Chennevière:

Qu'il fait gris ! Qu'il fait froid!

Une monotone pluie

A cousu le ciel aux toits.

Je pense aux pieds nus d'un enfant qui dort.

Les deux vers intermédiaires évoquent l'enchaînement (17), sans que son schéma ne soit précisé par aucun terme constitutif, ni dans l'énoncé lui-même, ni dans la période argumentative que constitue la strophe:

(17) il pleut de manière monotone alors le ciel est cousu aux toits

Quel schéma lui attacher? Comment l'interpréter argumentativement? On pensera à des schémas comme PLUIE DC NEG PERSPECTIVE OU MAUVAIS TEMPS DC DESTRUCTION, que l'on préfèrera au schéma PLUIE DC CouD, pourtant fondateur de (17). Je ferai I'hypothèse que la raison de cette préférence est que le schéma fondateur n'est pas préfiguré dans la signification de 
pluie, tandis que les deux autres le sont. Dans les termes de la Théorie des Blocs Sémantiques, le schéma fondateur de (17) n'est pas « doxal » tandis que les deux autres le sont. Nous préférons les interprétations doxales.

On pourrait s'attrister de ce conformisme mais on prendra garde à la définition que la théorie donne de la doxalité : il ne s'agit pas d'une fidélité aux croyances de la société mais d'une fidélité à la signification des mots (Carel 2017). Préférer les schémas PLUIE DC NEG PERSPECTIVE et MAUVAIS TEMPS DC DESTRUCTION, ce n'est jamais que préférer des schémas qui, inscrits ou préfigurés dans la signification du lexique (ici pluie et mauvais temps), peuvent être dits et par là même être mis en relation avec d'autres schémas qu'ils généralisent, spécifient, renforcent ou encore auxquels ils s'opposent : ce n'est jamais que préférer des schémas que l'on peut discuter, développer, objecter, en d'autres termes des schémas que l'on comprend. L'interprétation argumentative est guidée par les Idées que l'on comprend, les Idées que les mots isolés manifestent, les Idées du système de la langue, et non les Idées construites par assemblage de mots. Nous interprétons argumentativement au moyen des schémas présents sur la Carte Argumentative du Lexique. Comprendre, c'est recourir à des schémas doxaux.

J'insiste sur ce lien entre doxalité et compréhension car rien ne contraint à lire un texte de manière à le comprendre, de manière à pouvoir en parler. Les vers de Chennevière peuvent être lus comme concrétisant PLUIE DC COUD et, somme toute, certains poèmes ne donnent guère d'indice pour une interprétation argumentative doxale. Ce que je défends, c'est que de tels poèmes, associés à des schémas non doxaux, ne sont pas compréhensibles. Comprendre, c'est appréhender des schémas doxaux. Prenons ainsi le début de ce poème de Jean Sénac («Vieillesse », Les désordres) :

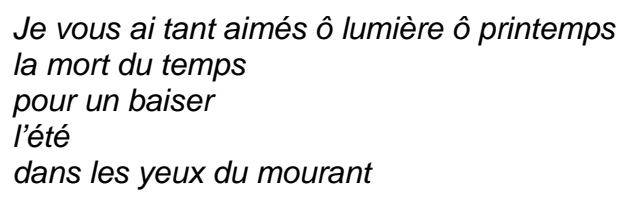

Maintenant le désir dynamite les carpes

les eaux troubles ferment le front

Pour déterminer le sens de l'avant dernier vers, on peut prendre modèle sur le second segment de (18), qui évoque (19):

(18) Ils ne trouvaient plus rien à se dire. Le mariage dynamite l'amour

(19) ils s'étaient mariés et du coup ils ne s'aimaient plus

compris, par exemple, comme concrétisant S'ENNUYER DC NEG S'AIMER. De manière parallèle, on associera alors l'avant-dernier vers de Sénac à l'enchaînement argumentatif (20):

(20) il y a du désir donc il n'y a plus de carpes Dans cette lecture (il y en a bien sûr d'autres), le verbe dynamiter est un simple lien argumentatif entre désir et carpes et, inversement, le nom carpes se voit donner de l'importance, à la manière du mot amour dans (18). Mais quel schéma doxal attacher à (20) ? Je n'en vois pas, et seule une interprétation par le schéma fondateur DÉSIR DC NEG CARPES s'impose à moi, de sorte que l'avant-dernier vers de Sénac reste pour moi incompréhensible. Je peux l'accepter, prendre plaisir au poème, comme on peut prendre plaisir à regarder de la peinture abstraite, quelques taches sur un fond uni. Mais je ne le comprends pas. Lorsqu'un locuteur construit un enchaînement, le lecteur, contraint à interpréter argumentativement, n'a que deux possibilités: ou choisir de lui attacher un aspect doxal ou accepter de ne pas comprendre.

Notons encore que cette contrainte de doxalité ne conduit pas à une et une seule interprétation argumentative. Le lecteur reste en partie libre. Le lecteur de Chennevière peut choisir aussi bien le schéma PLUIE DC NEG PERSPECTIVE que le schéma MAUVAIS TEMPS DC DESTRUCTION. II en va de même de la lecture du passage déjà cité du journal français $L e$ Monde :

(21) Le président veut renouer avec les corps intermédiaires, qui se sont sentis négligés sur le début de son quinquennat.

(21) évoque (22): 
(22) les corps intermédiaires se sont sentis négligés par lui donc le président veut renouer avec eux

que l'on peut comprendre comme concrétisant le schéma AVOIR NÉGLIGÉ DC DEVOIR FAIRE DES EFFORTS ou le schéma ETRE EN DANGER DC PRENDRE DES PRÉCAUTIONS : dans le premier cas le président serait décrit comme souhaitant garder des liens; dans le second, il est décrit comme contraint à garder ces liens. Les deux interprétations argumentatives sont possibles, les schémas étant tous les deux doxaux. Le lecteur reste libre.

Ainsi, la première contrainte qui guide l'interprétation argumentative est de mobiliser des schémas doxaux, c'est-à-dire des schémas qui se trouvent sur la Carte Argumentative du Lexique. Nous allons voir maintenant, c'est la deuxième contrainte, que pour déterminer le complexe argumentatif dans lequel se situe l'enchaînement évoqué il faut utiliser cette Carte. Le décodage argumentatif, nous l'avons vu en étudiant la période argumentative (14), la parcourt en allant du terme constitutif vers les schémas qu'il préfigure ou signifie. Nous allons voir que l'interprétation argumentative parcourt elle aussi la même Carte, mais en sens inverse, c'est-à-dire cette fois des schémas argumentatifs vers les mots. Pour montrer cela je m'appuierai sur un exemple, que j'ai souvent pris, celui du premier paragraphe de Claude Gueux, de Hugo :

(23) II y a sept ou huit ans, un homme nommé Claude Gueux, pauvre ouvrier, vivait à Paris. II avait avec lui une fille qui était sa maîtresse, et un enfant de cette fille. Je dis les choses comme elles sont, laissant le lecteur ramasser les moralités à mesure que les faits les sèment sur leur chemin. L'ouvrier était capable, habile, intelligent, fort maltraité par l'éducation, fort bien traité par la nature, ne sachant pas lire et sachant penser. Un hiver, l'ouvrage manqua. Pas de feu ni de pain dans le galetas. L'homme, la fille et l'enfant eurent froid et faim. L'homme vola. Je ne sais ce qu'il vola, je ne sais où il vola. Ce que je sais, c'est que de ce vol il résulta trois jours de pain et de feu pour la femme et pour l'enfant, et cinq ans de prison pour l'homme.

Comprendre ce paragraphe, c'est comprendre que, selon le locuteur, la société doit être transformée. Pour les rhétoriciens spécialistes de la persuasion, il s'agirait là d'un effet pragmatique. Le lecteur reconnaitrait un texte du XIXème siècle français, et, plus exactement encore, un texte de Hugo, et verrait alors dans ce récit une critique sociale: d'où il conclurait que, selon l'auteur, la société doit être améliorée. La langue serait pragmatiquement utilisée à la fin de persuader qu'il faut changer la Loi et la Justice. Le récit serait persuasif.

Une difficulté cependant se présente, c'est que ce texte date de 1834, bien loin des Misérables de 1862. Or Hugo est l'homme de son siècle ; il évolue politiquement au rythme de son siècle. En 1844, il est confident du roi Louis-Philippe; en juin 1848, il participe à la répression des émeutes ouvrières en dirigeant des troupes, face aux barricades; en décembre, il soutient la candidature à la présidence de Louis-Napoléon Bonaparte. Bref, le Hugo de 1834 n'est pas un écrivain naturaliste à la Zola. J'abandonnerai donc la solution pragmatique et défendrai que ce n'est pas l'auteur Hugo mais le narrateur de Claude Gueux qui communique la société doit être changée. Ce contenu implicite est sémantiquement communiqué, et non pas pragmatiquement. II est le résultat d'une interprétation argumentative du dernier énoncé du paragraphe.

Pour montrer cela, je remarquerai d'abord que cet énoncé évoque l'enchaînement argumentatif (24):

(24) son vol fournit trois jours de pain et de feu pour la femme et pour l'enfant et pourtant ce vol coûta cinq ans de prison pour l'homme

et cela sans qu'intervienne aucun terme constitutif. C'est l'opposition entre trois jours et cinq ans qui donne l'instruction de relier les deux propositions grammaticales par pourtant. Aucun terme constitutif n'est par contre présent et le lecteur doit argumentativement interpréter (24). On lui associera ainsi le schéma NEG FAUTE PT PUNITION, dans le nom duquel le symbole NEG signale la présence d'une négation argumentative, par exemple ne...pas mais aussi peu: ici le vol est décrit comme petit, Claude Gueux a peu volé.

Mais là ne s'arrête pas

l'interprétation argumentative, au sens technique que 
je cherche à donner à cette notion. Déterminer le sens d'un passage consiste, non seulement à attacher enchaînements argumentatifs et schémas, mais également à placer les enchaînements argumentatifs à l'intérieur de complexes argumentatifs. Mon hypothèse est que l'interprétation argumentative, comme le décodage argumentatif, recourt alors à la Carte Argumentative du Lexique, mais en la parcourant cette fois du schéma vers le mot :

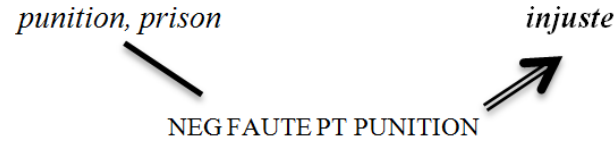

(24)
(25)

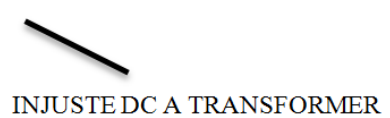

Le lecteur de (23) comprend qu'est évoqué (24), qu'il attache, conformément à la première règle que nous avons vue, à un schéma doxal: NEG FAUTE PT PUNITION. Puis, à partir du schéma concrétisé par (24), il remonte au mot injuste qui est choisi comme terme constitutif - un terme constitutif implicite. (24) est alors associé aux autres enchaînements évocables à partir du mot injuste, en particulier l'enchaînement (25) qui concrétise le schéma INJUSTE DC À TRANSFORMER:

(25) la société a été injuste avec Claude Gueux donc il faut la transformer

L'élément la société doit être transformée appartient au sens linguistique du paragraphe de Hugo. II est communiqué par le locuteur, cet être de discours que les énoncés se donnent comme responsable, et non par le sujet parlant. La famille d'enchaînements dans laquelle la paraphrase d'un énoncé se situe peut être explicite, comme dans le cas de la période argumentative (14), mais elle peut être en partie implicite, comme ici. Donner un sens à un énoncé, c'est déterminer les enchaînements qu'il évoque explicitement, les attacher à des schémas, et les regrouper avec les enchaînements argumentatifs du même complexe. C'est déterminer le terme constitutif, qu'il soit explicite, comme dans les décodages argumentatifs, ou qu'il soit implicite, comme lorsqu'il y a interprétation argumentative.

On peut reformuler cela autrement et remarquer que les mots d'un énoncé peuvent être argumentativement importants de deux manières. Soit ils expriment leur signification argumentative, fournissent la structure et le sens des enchaînements évoqués, et les regroupent en un unique complexe : ils sont alors constitutifs et servent le décodage argumentatif. Mais ils peuvent également être reliés en enchaînement argumentatif grâce à la syntaxe ou aux conjonctions, comme ici dans le passage de Claude Gueux, et faire alors seulement allusion au schéma qu'ils concrétisent et au terme dont ils développent la signification. La Carte Argumentative du Lexique est dans ce cas parcourue en sens inverse, le terme constitutif est implicite, et il y a interprétation argumentative.

Ce double rôle des termes, organisateurs ou organisés, cette double lecture, argumentativement décodante ou argumentativement interprétative, cette alternative découle du fait, fondamental pour le structuralisme, que les mots renvoient aux mots. Un mot peut ainsi signifier d'autres mots - c'est le décodage argumentatif -, mais il peut également être signifié par d'autres mots et il y a alors interprétation argumentative. Le renvoi des mots aux mots peut s'effectuer dans un sens comme dans l'autre. Tout autant que le décodage, l'interprétation argumentative est un phénomène sémantique. Nous l'avons vu à partir de l'exemple du premier paragraphe de Claude Gueux: c'est sémantiquement et non pragmatiquement qu'est évoqué la société a été injuste avec Claude Gueux. Nous allons le revoir à propos d'un autre cas : l'emploi de mais.

\section{L'interprétation dans la langue : retour sur mais}

On se souvient qu'Anscombre et Ducrot se sont appuyés sur leur description de mais pour défendre que l'argumentation est dans la langue. En effet, selon eux, tout emploi de $A$ mais $B$ rejette une argumentation $A$ donc $r$ issue de A. Autrement dit, la signification même de mais contient l'instruction «trouver l'enchaînement $A$ donc $r$ rejeté par le locuteur » et contient ainsi une allusion au fait que le 
segment A évoque une argumentation. II en va de même de presque, dont la signification indique que presque $X$ sert les mêmes conclusions que $X$, ou de à peine, dont la signification indique, à l'inverse, que à peine $X$ argumente comme non- $X$. Tous ces « mots du discours » contiennent, à l'intérieur de leur signification, des indications quant au potentiel argumentatif de l'énoncé dans lequel ils apparaissent, et la langue, qui les contient, possède donc, parmi ses concepts, le fait qu'un segment peut servir une conclusion. Argumenter n'est pas un acte perlocutoire, s'ajoutant à une information qui, elle seule, serait illocutoire. L'argumentation est dans la langue. Je me propose ici d'utiliser le même procédé qu'Anscombre et Ducrot pour montrer cette fois que l'interprétation argumentative est dans la langue.

Repartons de la description d'Anscombre et Ducrot (1977), selon laquelle l'emploi de $A$ mais $B$, celui que se traduit en espagnol par pero et non par sino, rejette un enchaînement argumentatif $A$ donc $r$. On se souvient de l'exemple de base. II s'agit de (26), utilisé pour refuser une promenade:

(26) il fait beau mais je suis fatigué

Selon Anscombre et Ducrot, $A=i l$ fait beau évoque l'enchaînement argumentatif $A$ donc $r$ :

\section{il fait beau donc la promenade serait agrable}

et le locuteur de (26) rejette cet enchaînement au moyen de $\mathrm{B}=j e$ suis fatigué. Je me propose de montrer qu'il n'en va pas toujours ainsi. L'élément évoqué par A et rejeté par B n'a pas toujours la forme A donc r. II s'agit parfois d'un enchaînement argumentatif dont le premier segment n'est pas identique à $A$.

L'exemple est le suivant. Imaginons une institutrice, mécontente du fonctionnement de l'école de la petite ville où elle travaille. Elle a le sentiment que ses collègues sont moins sérieux qu'elle, elle a le sentiment que sa propre valeur n'est pas reconnue. Elle se plaint tout particulièrement d'un de ses collègues, Monsieur Laval, qui n'est jamais chapitré par le directeur:

(27) le travail du fils Martin n'a pas été sanctionné, malgré les fautes d'orthographe
(A), mais le directeur n'a bien sûr pas chapitré Monsieur Laval (B)

Quel est l'élément, évoqué par A, que B rejette ici ? S'appuyant sur les analyses d'Anscombre et Ducrot, on pourrait d'abord penser qu'est rejeté l'enchaînement (28), qui a la forme $A$ donc $r$ attendue:

(28) le travail du fils Martin n'a pas été sanctionné, malgré les fautes d'orthographe, donc le directeur a chapitré Monsieur Laval

On notera cependant que ce n'est pas l'absence de mauvaise note, par elle-même, qui est un argument pour l'intervention du directeur; (28) ne repose pas sur l'idée que le directeur souhaite les mauvaises notes et est déçu des bonnes. L'absence de mauvaise note manifeste le laxisme de Monsieur Laval et c'est ce laxime de Monsieur Laval qui est un argument pour l'intervention du directeur. Autrement dit, ce n'est pas (28) mais (29) qui est rejeté:

(29) Monsieur Laval a été laxiste avec le fils Martin donc le directeur l'a chapitré

Or (29) ne comporte par A à titre de segment. Comment rendre compte de cela? Comment élargir la description d'Anscombre et Ducrot pour qu'elle englobe notre exemple (27)?

Pour répondre à ce problème, j'attacherai à $A$ mais $B$, non pas l'instruction restreinte «trouver l'enchaînement $A$ donc $r$ rejeté par le locuteur » mais l'instruction élargie "trouver l'enchaînement communiqué par $\mathrm{A}$ et rejeté par le locuteur » et je dirai qu'un enchaînement est « communiqué » lorsqu'il est évoqué par décodage ou par interprétation argumentative. Dans le cas de (26) il fait beau mais je suis fatigué, l'enchaînement rejeté il fait beau donc la promenade serait agréable découle d'un décodage de A : le terme constitutif est faire beau, sa signification préfigure BEAU TEMPS DC ACTIVITÉ EXTÉRIEURE AGRÉABLE et A communique il fait beau donc la promenade serait agréable, enchaînement rejeté par je suis fatigué. Dans le cas de (27), l'enchaînement rejeté est (29):

(27) le travail du fils Martin n'a pas été sanctionné, malgré les fautes d'orthographe, mais le directeur n'a bien sûr pas chapitré Monsieur Laval 
(29) Monsieur Laval a été laxiste avec le fils Martin donc le directeur l'a chapitré

Il découle cette fois d'une interprétation de A. Par son emploi de malgré, le segment A évoque l'enchaînement (30):

(30) le fils Martin fait des fautes d'orthographes pourtant Monsieur Laval ne l'a pas sanctionné

enchaînement qui, construit grammaticalement, apparaît donc comme devant être interprété. (30) est alors attaché au schéma FAUTE PT NEG PUNITION, qui est lui-même relié au terme constitutif implicite laxiste :

faute

FAUTE PT NEG PUNITION

(30) est placé dans le même complexe argumentatif que (29). A, qui évoque (30) explicitement, évoque ainsi (29) implicitement. A «communique» par interprétation argumentative (29) et (29) est rejeté par B.

La compréhension de (31) reposerait sur le même mécanisme :

(31) le travail du fils Martin n'a pas été santionné, malgré les fautes d'orthographe, mais le gamin est quand même très en colère contre son instituteur

Le premier segment $A$ évoque à nouveau (30):

(30) le fils Martin fait des fautes d'orthographes pourtant Monsieur Laval ne l'a pas sanctionné

qui est à nouveau attaché à FAUTE PT NEG PUNITION. Mais cette fois, le terme constitutif implicite est indulgent de sorte qu'est implicitement évoqué par A l'enchaînement (32):

(32) Monsieur Laval a été indulgent avec le fils Martin donc ce dernier n'est pas en colère contre lui

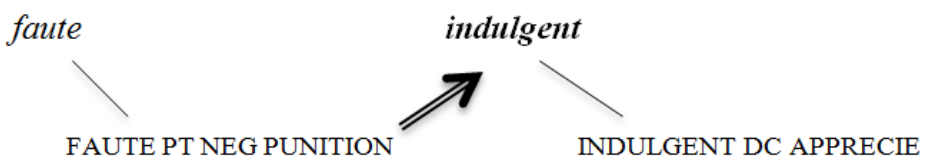

(30)
C'est l'enchaînement (32) qui est rejeté par B. A nouveau A se contente de "communiquer" l'enchaînement évoqué. II n'en est pas le premier segment.

Nous pouvons maintenant revenir à notre propos général : celui de montrer que l'interprétation argumentative est dans la langue. Faisant allusion à ce que $A$ «communique », et non pas simplement à ce que A permet de conclure, la signification de mais contient des indications sur le phénomène d'interprétation argumentative. La langue, qui comporte mais, contient donc, parmi ses concepts, celui d'interprétation argumentative. L'interprétation n'est pas un phénomène second, s'appuyant sur un phénomène linguistique premier d'information. L'interprétation argumentative est dans la langue, au même titre que le décodage argumentatif.

Un dernier exemple. II a été fait abstration, dans ce qui précède, de ce que l'enchaînement communiqué par $A$ et rejeté par $B$ pourrait ne pas provenir de la signification des mots de A, mais leur être seulement contextuellement associé. II n'y aurait alors, ni décodage argumentatif, ni interprétation argumentative de A. On peut penser à des emplois de mais comme (33):

(33) elle est belle mais elle est intelligente qui semble rejeter elle est belle donc elle n'est pas intelligente : or cet enchaînement n'est pas préfiguré dans la signification de belle, et n'est donc pas décodé. Nous allons voir que l'exemple peut cependant être expliqué en tenant compte, comme je le propose, de l'interprétation argumentative.

Certes, une difficulté apparaît de prime abord. Elle tient au fait que le premier segment de (33) n'appelle pas, de lui-même, une interprétation argumentative. Contrairement au premier segment de (27), il contient un terme constitutif, le terme belle, qui signifie le schéma argumentatif NEG RÈGLE POUR APPRÉCIER PT APPRECIÉ UNIVERSELLEMENT de sorte qu'est évoqué par A l'enchaînement (35):

(35) il n'y a pas de règle pour apprécier le physique pourtant son physique est apprécié universllement 
compris comme concrétisant NEG RÈGLE POUR APPRÉCIER PT APPRECIÉ UNIVERSELLEMENT et que cet enchaînement est placé dans le complexe argumentatif défini par belle. J'admettrai que l'emploi de mais intelligente lance une interprétation argumentative de (35) de manière à le situer dans un nouveau complexe argumentatif. Dans la mesure où belle ne préfigure pas BELLE DC NEG INTELLIGENTE, il est fait appel à un nouveau terme $X$ qui, englobant (35), signifie cette fois $X$ DC NEG INTELLIGENTE. Le français contemporain en fournit un, le terme blonde qui a pris le même sens que la collocation « jolie idiote ». Ainsi, l'exemple (33) ne rejette pas l'enchaînement elle est belle donc elle n'est pas intelligente, qui est absent de la signification de belle; l'exemple (33) rejette l'enchaînement (36):

(36) elle est blonde donc elle n'est pas intelligente,

qui est seulement «communiqué » par belle, après interprétation.

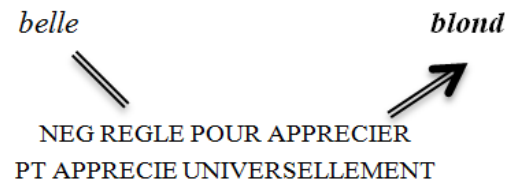

(35) BLONDE DC NEG INTELLIGENTE

D'autres emplois de mais sont du même type, par exemple (37) qui rejette, non pas (38), mais (39) - on pensera à un petit garçon:

(37) il fait semblant d'être un cow-boy mais cela ne l'amuse pas

(38) il fait semblant d'être un cow-boy donc il s'amuse

(39) il joue donc il s'amuse

conformément à la Carte Argumentative du Lexique:
(41) même s'il s'agit de civils, nous les bombarderons

selon la Carte Argumentative du Lexique:

être sous le tir, menacé
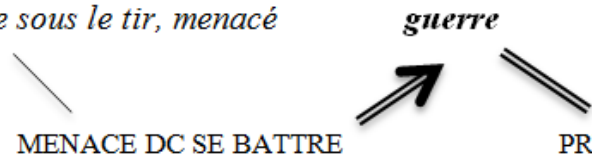

PRECIEUX PT DETRUIRE

(41)

Notre compréhension est à nouveau guidée par la Carte Argumentative du Lexique, comme lorsqu'il s'agissait, nous l'avons vu dans la deuxième partie, de comprendre des enchaînements argumentatifs qui ont été grammaticalement construits. Le recours à la Carte est le même, ainsi que ses difficultés : comme l'énoncé le désir dynamite les carpes, le discours il ressentait du désir mais sans dynamiter les carpes est impossible à comprendre.

On objectera à cette description de (33) la version masculine (40):

(40) il est beau mais intelligent

En effet, le français ne comporte pas de correspondant masculin à blonde, ni même de collocation «joli idiot». Par quel terme $X$ interpréter l'enchaînement (41) d'abord décodé grâce au terme beau?

(41) il n'y a pas de règle pour apprécier le physique pourtant son physique est apprécié universllement

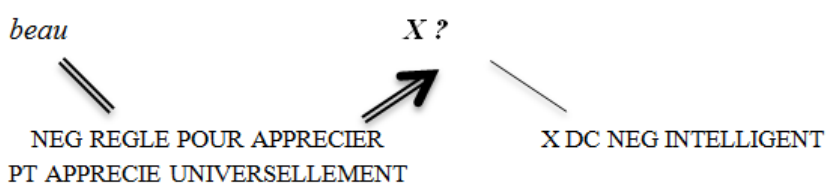

(41) faire semblant

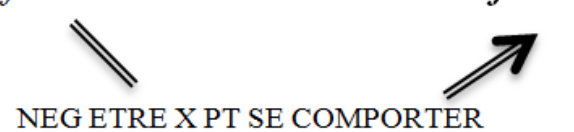
COMME UN X

De même encore, (40) rejette un enchaînement comme (41):

(40) nous sommes sous le tir de leurs canons mais nous ne bombarderons pas leurs civils
Cet emploi de mais serait encore différent. On notera JOUER DC S'AMUSER cependant le clin d'œil, féministe : (40) est compris par allusion intertextuelle à la version féminine (33). Son sens ne se calcule pas à partir du mot beau mais découle d'une mise au masculin de ce que communique (33). II en est directement le masculin grammatical. (33) excluait elle est blonde donc elle 
n'est pas intelligente; (40) exclut la version masculine il est blond donc il n'est pas intelligent, sans passer par la signification de beau, et dévoile ce faisant la dissymétrie de beau et de belle.

Notre compréhension des textes s'appuie, systématiquement, sur la Carte Argumentative du Lexique.

\section{Conclusion}

La Théorie des Blocs Sémantiques donne un sens argumentatif à l'hypothèse structuraliste selon laquelle les mots renvoient aux mots. Il ne s'agit plus de dire que les mots se définissent les uns les autres par opposition, mais qu'ils entrent dans des

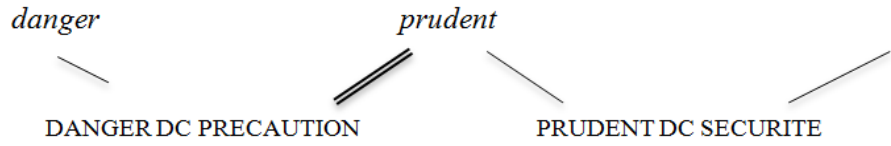

relations de paraphrase argumentative. Le mot danger est ainsi relié au mot prudent car Pierre a été prudent se paraphrase par il y avait un danger et donc Pierre a pris des précautions; et le mot prudent est relié au mot sécurité car Pierre est prudent peut également évoquer Pierre est prudent sur la route donc Marie est en sécurité avec lui :

La Théorie des Blocs Sémantiques place cette Carte Argumentative du Lexique au centre de notre compréhension des textes.

Les mots d'un texte peuvent en effet avoir deux rôles argumentatifs. Ils peuvent exprimer leur signification et guider de cette manière la paraphrase argumentative en donnant la structure des enchaînements évoqués et en regroupant ces enchaînements à l'intérieur d'un même complexe argumentatif. La Carte est alors parcourue des mots vers les schémas argumentatifs; il y a décodage argumentatif. Mais les mots peuvent également être directement reliés en enchaînements grâce à la syntaxe ou à des mots outils comme malgré, si, donc. La Carte est alors parcourue dans l'autre sens. L'enchaînement est associé à un schéma de la Carte (un schémas doxal) puis ce schéma est relié à un mot, plaçant l'enchaînement à l'intérieur d'un complexe argumentatif. II y a alors interprétation argumentative. Les sémantiques compositionnelles favorisent le décodage pour favoriser le calcul: les mots ont une signification et cette signification serait systématiquement utilisée pour calculer celle des

groupe de mots. J'ai essayé de montrer que prendre en compte le phénomène d'interprétation argumentative n'est pas abandonner le projet d'un calcul du sens. L'interprétation, en partie libre, est tout de même guidée par la Carte Argumentative du Lexique.

sécurité Nous lisons en décodant et en interprétant. Le premier procédé demande seulement de connaître la signification des mots ; le second demande par contre de connaître l'entièreté des schémas doxaux et avec eux les divers mots qui les signifient. II repose sur une connaissance complète de la langue, qui, si elle fait défaut (je pense aux apprenants, enfants ou adultes), ralentira, voire empêchera, la lecture. Notre compréhension dépend de notre connaissance de la Carte Argumentative du Lexique.

\section{Références}

Anscombre J.-Cl. et O. Ducrot (1977) "Deux mais en français", Lingua, 43, 23-40.

Carel, M. (2017) "Significaçao e argumentaçao », Signo, UNISC, vol 42, $\mathrm{n}^{\circ} 73,2-20$.

Christopulos, G. (2018) «Au delà de l'isotopie », SHS Web of Conferences, vol 46, article $n^{\circ} 06004,6^{\text {ème }}$ Congrès Mondial de Linguistique Française. https://doi.org/10.1051/shsconf/20184606004

Lescano, A. (2005) «Lorsque très ne renforce pas », Revue Romane, n40, vol.1, 101-114. 
Lescano, A. (2008). Vers une grammaire argumentative de la phrase: le cas de l'article défini et indéfini en français et en espagno, thèse de doctorat en Sciences du Langage (EHESS).

Saenz Suarez, C. (2019) «Autour du mot spectacle. L'hypothèse de la résonance ». Mémoire de master de l'EHESS (Paris).

\section{COMO CITAR ESSE ARTIGO}

CAREL, Marion. Interprétation et décodage argumentatifs. Signo, Santa Cruz do Sul, v. 44, n. 80, ago. 2019. ISSN 1982-2014. Disponível em: <https://online.unisc.br/seer/index.php/signo/article/view/13661>. Acesso em: doi: https://doi.org/10.17058/signo.v44i80.13661. 